\title{
DIFERENCIAS ENTRE ACTORES SOCIALES EN EL CONOCIMIENTO Y LA PERCEPCIÓN DE LA VEGETACIÓN DE LA CUENCA DE LA CIÉNAGA DE LA VIRGEN (CARTAGENA DE INDIAS, COLOMBIA)
}

\section{DIFFERENCES IN SOCIAL ACTORS' KNOWLEDGE AND PERCEPTION OF THE VEGETATION OF THE BASIN IN THE CIÉNAGA DE LA VIRGEN (CARTAGENA DE INDIAS, COLOMBIA) VEGETATION}

\author{
Edilbert Torregroza Fuentes ${ }^{1}$, Jorge Llamas Chávez ${ }^{2}$ y Francisco Borja Barrera ${ }^{3}$
}

\begin{abstract}
Resumen
La percepción y las actitudes de los actores sociales presentes en un determinado territorio son vitales para su adecuada gestión. Tales aspectos fueron evaluados para el caso cuenca de la Ciénaga de la Virgen (Cartagena de Indias, Colombia), en relación a doce especies vegetales presentes en el territorio, considerando la cuenca desde un enfoque socioecosistémico, desde el que se recalca la interacción y adaptación entre los sistemas sociales y ecológicos. El estudio aborda aspectos como el conocimiento de una determinada flora del lugar, la percepción sobre posibles beneficios o perjuicios derivados de aquella, y las diferencias entre especies nativas y especies introducidas. Metodológicamente el trabajo correspondió a una investigación de carácter cuantitativo con aplicación de una encuesta de percepción a cuatro grupos de actores dentro de la trama social. El trabajo permitió comprender cómo, en función de su rol, los individuos valoran de manera propia el papel de determinadas especies, en tanto que agentes biológicos claves.
\end{abstract}

Palabras clave: Rol social, plantas, socioecosistema.

\begin{abstract}
Perception and attitude of social actors in a territory are vital for their proper management. Such aspects were evaluated in the case of the basin of the Ciénaga de la Virgen (Cartagena de Indias, Colombia) in relation to some plants that are present in the territory, considering the basin from the viewpoint of the socio-ecological system. This approach emphasizes the interaction and adaptation amongst social and ecological systems. The study addresses issues such as knowledge of the local vegetation, perception of potential benefits or damage resulting from them, and differences between both native and introduced species in the territory. In terms of methodology, the work is mostly of a quantitative research with the application of a perception survey to four main social groups. The results allowed a comprehension of the way in which individuals, based on their social role, perceive and value plant species as key biological agents.
\end{abstract}

Key words: Social perception, plants, social-ecological systems.

\section{Introducción.}

El conocimiento y la percepción de la población acerca del medio natural $y$, en particular, acerca de algunos de sus componentes, son aspectos de una gran importancia para la gestión integral de un territorio y, especialmente, para la conservación y el uso sostenible de su diversidad biológica (Callaghan et al., 2004). Está claro que la percepción no es una temática nueva ni sobre la que abunde el consenso (Lynch, 1960; Lowenthal, 1961). Hace ya décadas que quedó establecida la relación entre los sentidos humanos, la percepción y el conocimiento, atribuyéndose al ejercicio de aquellos un papel fundamental en la definición de la percepción (Warnock, 1974). Según Capel (1973), el papel de la percepción humana es decisivo en la formación de una imagen del medio real, la cual, y no éste, es la que influye directamente sobre su comportamiento. Con el tiempo se tiene cada vez más en cuenta que la percepción y la actitud de las personas constituyen un elemento necesario para la completa comprensión de un sitio (Fleming, 1975). Gibson (1980), por su parte, desde la psicología ambiental, al referirse al estudio de la percepción ambiental, la consideraba como el resultado de la experiencia humana a partir de las sensaciones. De tal manera que la percepción se concebiría como un producto elaborado por los seres humanos, en donde toman significado los objetos y el espacio con el interactúan (Conroy, 2002). Se logra comprender así que la relación existente entre los seres humanos y su entorno natural es, en gran medida, consecuencia de su percepción ambiental (Lefebvre, 1991). 
Recientemente ha venido señalándose que la percepción ambiental puede entenderse como la manera en que cada individuo o cada colectivo aprecian su entorno. Esta valoración tiene una incidencia significativa en la toma de decisiones de alcance medioambiental y en el tipo de relación que los seres humanos establecen con su entorno (Fernández, 2008). Ahora bien, los pobladores de un determinado territorio pueden tener diversos niveles de percepción y conocimiento, en general en lo que atañe al entorno que le rodea, y particularmente acerca de su componente vegetal. De lo que se deriva que sobre determinadas especies de plantas se lleven a cabo desiguales tipos de acercamiento o gestión, dependiendo en parte del grupo social del que se trate (ciudadanos en general, académicos, miembros del sector empresarial, tomadores de decisiones a nivel gubernamental...), los cuales dan a sus actuaciones un sentido específico, consciente o inconscientemente. Los actores sociales suelen verse movidos por representaciones y valores, muchos de ellos de carácter simbólico, derivados de su relación con el entorno. Entre estos elementos pueden considerarse determinadas especies vegetales, de las que tales agentes sociales pueden obtener algún tipo de beneficio o por las que pueden verse perjudicados, acorde con sus proyecciones, necesidades, tradiciones, etc. (Quétier et al., 2007). No obstante, no es mucho lo que se ha explorado acerca del comportamiento de los diferentes agentes sociales cuando se disputan algunos de estos elementos (O'Brien \& Leichenko, 2003), o cuando entran en conflicto por la apropiación de determinados servicios ecosistémicos (trade-offs). Tal situación bien podría plantearse con respecto al conocimiento y las actitudes de determinados colectivos frente al potencial de las especies vegetales presentes en un territorio.

Además de valorar la importancia de la percepción del entorno natural por parte de los actores sociales, el presente estudio enfoca la cuenca de la Ciénaga de la Virgen en tanto que socioecosistema, un concepto que promueve el análisis y la gestión integral del territorio definido a partir de la interrelación establecida entre los seres humanos y su entorno natural, y desde el que se insiste en que la delimitación entre los sistemas ecológicos y sociales es artificial o, cuando menos, arbitraria (Berkes \& Folke, 1998). Nuestra investigación aplica tal enfoque centrándose en el caso del entramado natural y social asociado a la Ciénaga de la Virgen y su cuenca, cuyo principal asentamiento humano corresponde al distrito de Cartagena de Indias (Colombia). Asimismo, partimos de la idea de que las especies vegetales de un determinado socioecosistema tienen un gran peso relativo en la imagen mental que los colectivos humanos elaboran de su entorno natural, dado que constituyen un elemento básico del mismo, en su categoría de agente biológico destacado (Anderies et al., 2006). Este hecho adquiere su verdadera dimensión si se considera que las potencialidades de la cubierta vegetal, entendidas en términos de beneficios sociales, son un aspecto fundamental para estimar los servicios ecosistémicos que dicho territorio puede ofrecer a sus pobladores (Millennium Ecosystem Assessment, 2005). De este modo, se ha venido señalando que los ecosistemas urbanos, entidades que privilegian el desenvolvimiento humano, se caracterizan por la existencia de actores o grupos sociales que se mueven en función de la ganancia, el poder, la necesidad, y también del conocimiento que poseen de sus diversos componentes. Pero dichos actores son categorías heterogéneas, que aunque se definan por el rasgo fundamental que los identifica, pueden adoptar posiciones diversas dentro del entramado social. Se trataría, así pues, de sujetos, individuales o colectivos, cuyo comportamiento — incluyendo preferencias, actitudes y conocimientos - determinan los procesos claves a escala local. En este sentido suelen identificarse actores asociados a la lógica económica o empresarial, a la del conocimiento científico, así como agentes políticos relacionados con la toma de decisiones en el gobierno local, o sujetos individuales en tanto que ciudadanos relacionados vitalmente con el territorio (Pírez, 1995).

Finalmente, hablamos de vegetación para referirnos al conjunto de plantas que integran un determinado sistema ecológico (Matteucci \& Colma, 1998). El tipo y el grado de cobertura de una determinada formación vegetal denuncian las condiciones climáticas de un lugar, siendo también un buen indicador de la clase y la situación de los suelos. Las formaciones vegetales suelen incluir tipos diversos de plantas que resultan percibidas en mayor o menor medida y de forma muy diferente por los pobladores dependiendo de cada sitio. Que los pobladores se percaten de tal diversidad viene condicionado por el significado que cada especie tenga para los mismos, ya sea por su utilidad ornamental, alimenticia, comercial o cultural, ya sea por la convivencia deseable o no con estos componentes de la biosfera, con los cuales, en última instancia, una gran parte de los seres humanos comparten su espacio vivido. De igual manera, no podemos perder de vista que la vegetación actual de un determinado lugar es, en mayor o menor medida, el resultado de la acción humana sobre el medio natural. Las formaciones vegetales son, por tanto, uno de los elementos más conspicuos del paisaje y de más fácil percepción por el ser humano. Además, las especies que las integran responden individual o conjuntamente a los factores ecológicos, por lo cual reflejan muchas de sus características ambientales (Matteucci \& Colma, 1998). De ahí que la percepción social de la vegetación constituya un aspecto sumamente importante en términos de gestión, pues es capaz de manifestar el valor diferencial que los habitantes de un 


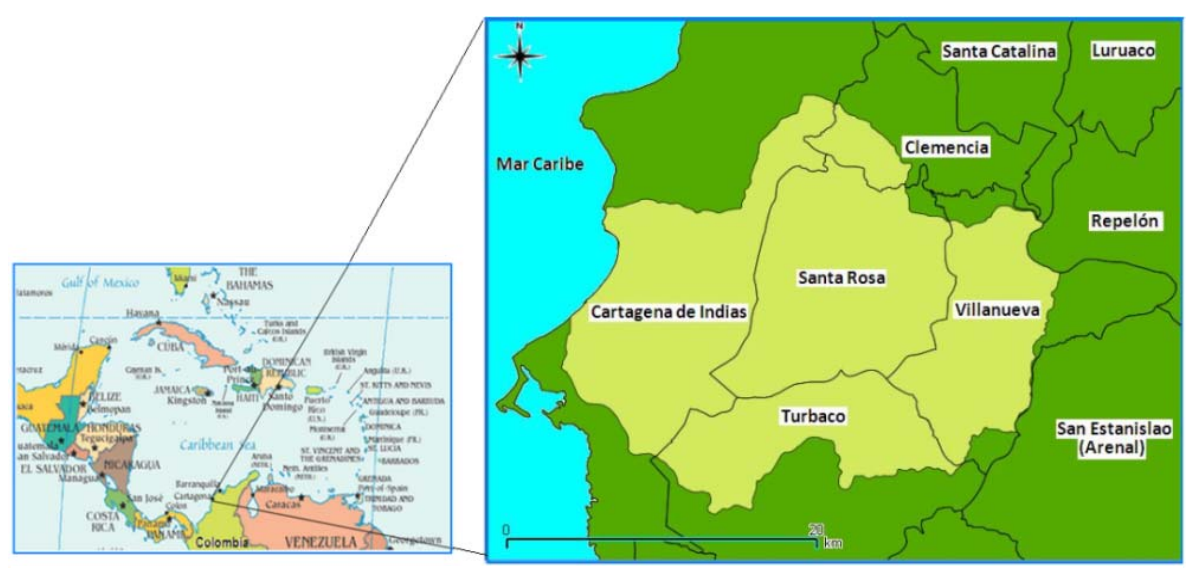

Figura 1. Localización de la Cuenca de la Ciénaga de la Virgen en el contexto administrativo del sector septentrional del Departamento de Bolívar.

Fuente: Elaboración propia.

lugar otorgan a cada especie, e incluso, en determinados contextos, a cada individuo (e.g. árboltótem). Creemos, por tanto, que a partir del análisis de la percepción de las especies vegetales puede evidenciarse el nivel de identificación, preferencia y afectividad social, e incluso llevarse a cabo una valoración dentro del paisaje general de la cuenca de la Ciénaga de la Virgen.

Desde este punto de vista, el presente trabajo pretende, primeramente, concretar las diferencias existentes en el conocimiento y la percepción de los diferentes actores sociales presentes en el entorno de la Ciénaga de la Virgen, en relación a una serie de especies vegetales representativas del lugar; y, en segundo término, plantear cómo este conocimiento y esta percepción pueden ayudar a identificar criterios válidos para una gestión integral del territorio.

\section{Área de estudio.}

Como se indicó con anterioridad, el ámbito de referencia de la presente investigación se corresponde con la cuenca vertiente de la Ciénaga de la Virgen
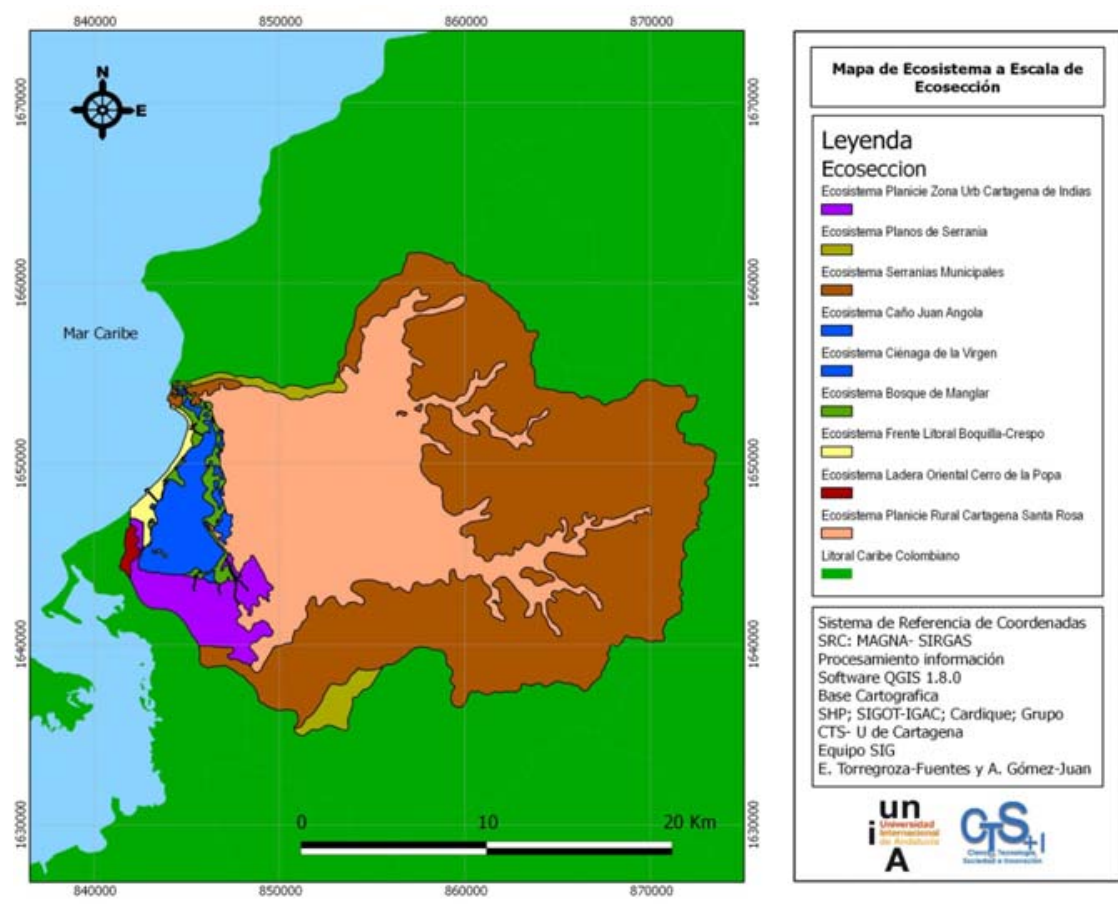

Figura 2. Identificación y delimitación de los ecosistemas de la Cuenca de la Ciénaga de La Virgen a escala de ecosección. Fuente: Torregroza, E. (en preparación). 
Tabla 1. Síntesis climática del entorno de la Ciénaga de la Virgen.

\begin{tabular}{|c|c|c|c|}
\hline Clima & Tropical & cálido & \\
\hline \multirow{3}{*}{$\begin{array}{l}\text { Precipitación } \\
\text { (Promedio anual) }\end{array}$} & \multirow{3}{*}{$976 \mathrm{~mm}$} & diciembre - abril & Seco \\
\hline & & mayo - agosto & Transición \\
\hline & & septiembre - noviembre & Lluvioso \\
\hline $\begin{array}{l}\text { Temperatura } \\
\text { (Promedio anual) }\end{array}$ & \multicolumn{3}{|l|}{$28^{\circ} \mathrm{C}$} \\
\hline Humedad relativa & \multicolumn{3}{|c|}{ Superior al $90 \%$} \\
\hline $\begin{array}{l}\text { Velocidad del Viento } \\
\text { (Promedio anual) }\end{array}$ & \multicolumn{3}{|c|}{$3.18 \mathrm{~m} / \mathrm{s}$ (brisa suave o moderada) } \\
\hline $\begin{array}{l}\text { Evapotranspiración } \\
\text { (media anual) }\end{array}$ & \multicolumn{3}{|c|}{$1734.32 \mathrm{~mm}$} \\
\hline
\end{tabular}

Fuente: Elaboración propia a partir de datos IDEAM e IGAC.

(CCV), la cual se localiza en la parte central del frente caribeño del Departamento de Bolívar, abarcando unos $520 \mathrm{~km}^{2}$ de extensión. La Ciénaga en sí es una laguna costera de más de $20 \mathrm{Km}^{2}$ de superficie y una profundidad media cercana a $1.5 \mathrm{~m}$, la cual queda separada del Atlántico por una barrera arenosa de unos $10 \mathrm{~km}$ de longitud permeabilizada en varios puntos. Este humedal recibe las aguas de un piedemonte en forma de circo que salva un desnivel de en torno a $200 \mathrm{~m}$. Desde el punto de vista políticoadministrativo la CCV ocupa parte de las municipalidades de Cartagena de Indias, Turbaco, Villanueva, Santa Rosa de Lima y Clemencia (Figura 1). Sin embargo, desde el punto de vista ecosistémico, no es esta la demarcación más adecuada para el análisis territorial, sino aquella otra que identifica unidades funcionales a distintas escalas espaciales (regionalización ecológica), las cuales se expresan en dimensiones que, por regla general, no se compadecen con las fronteras administrativas (Borja et al., 2009). En tal sentido, se ha llevado a cabo una propuesta de regionalización ecológica de la CCV (Torregroza, en preparación; Torregroza et al., en prensa), donde se han distinguido diez ecosistemas a escala de ecosección (Figura 2).

Desde el punto de vista climático, en la Tabla 1 se incluyen los valores de los principales parámetros de la zona estudiada, los cuales nos permiten clasificarla como un ámbito perteneciente a un clima de tipo Tropical cálido, caracterizado por una precipitación anual cercana a los 1000 $\mathrm{mm}$ que se concentran en los meses de septiembre a noviembre, así como por un promedio térmico en torno a los $28^{\circ} \mathrm{C}$.
Por su parte (Tabla 2), la vegetación asociada a la cuenca en estudio muestra una gran variedad, no sólo condicionada por las características climáticas, edáficas, topográficas, etc. (vegetación psammófila, haloxihidrófila, xerofítica, semixerofítica), sino además merced a las complejas interacciones derivadas del uso y los aprovechamientos ejercidos por los habitantes de la cuenca.

\section{Metodología.}

El levantamiento de datos para la presente investigación se llevó a cabo realizando una encuesta mediante el método de la entrevista personal. Para ello se elaboró un cuestionario general en el que se abordaron aspectos relativos al conocimiento y la percepción social de una serie de 12 especies vegetales seleccionadas ex profeso. Las encuestas fueron practicadas entre

Tabla 2. Vegetación representativa de la CCV.

\begin{tabular}{|c|c|c|}
\hline $\begin{array}{l}\text { Tipo } \\
\text { vegetación }\end{array}$ & $\begin{array}{l}\text { Ubicación } \\
\text { básica }\end{array}$ & Nombre científico \\
\hline $\begin{array}{l}\text { Vegetación } \\
\text { Psammófila }\end{array}$ & $\begin{array}{l}\text { Zona litoral - } \\
\text { Planicie Zonas } \\
\text { arenosas }\end{array}$ & $\begin{array}{l}\text { Coccoloba uvifera L. } \\
\text { Capparis odoratissima Jacq. } \\
\text { Acacia farnesiana (L.) Willd. } \\
\text { Cocos nucifera L }\end{array}$ \\
\hline $\begin{array}{l}\text { Vegetación } \\
\text { Haloxihidrófila }\end{array}$ & $\begin{array}{l}\text { Zona de } \\
\text { humedales } \\
\text { Planicie Zonas } \\
\text { de salinas y } \\
\text { cenagosas }\end{array}$ & $\begin{array}{l}\text { Rhizophora mangle L. } \\
\text { Avicennia germinans (L.) L. } \\
\text { Conocarpus erecta L. } \\
\text { Laguncularia racemosa C.F.Gaertn. } \\
\text { Portulaca oleracea L } \\
\text { Prosopis juliflora DC. }\end{array}$ \\
\hline Vegetación & $\begin{array}{l}\text { Zona litoral - } \\
\text { Planicie }\end{array}$ & $\begin{array}{l}\text { Pithecellobium Mart. } \\
\text { Pereskia guamacho F.A.C.Weber } \\
\text { Acacia farnesiana (L.) Willd. }\end{array}$ \\
\hline Xerofítica & $\begin{array}{l}\text { Zonas muy } \\
\text { secas }\end{array}$ & $\begin{array}{l}\text { Opuntia wentiana Britton \& Rose } \\
\text { Cordia dentata Poir. } \\
\text { Crescentia cujete L. }\end{array}$ \\
\hline $\begin{array}{l}\text { Vegetación } \\
\text { Semixerofítica }\end{array}$ & $\begin{array}{l}\text { Zonas de } \\
\text { fuerte presión } \\
\text { antrópica - } \\
\text { Relictos de } \\
\text { bosques }\end{array}$ & $\begin{array}{l}\text { Cnidoscolus tubulosus I.M.Johnst. } \\
\text { Acacia farnesiana (L.) Willd. } \\
\text { Astronium graveolens Jacq.- } \\
\text { Libidibia coriaria (Jacq.) Schltdl. } \\
\text { Pithecellobium saman (Jacq.) Benth. } \\
\text { Spondias mombin L. } \\
\text { _Gliricidia sepium (Jacq.) Kunth_- } \\
\text { Tabebuia Gomez } \\
\text { Ceiba pentandra (L.) Gaertn. } \\
\text { Guaiacum L. } \\
\text { Roseodendron chryseum (Blake) Miranda } \\
\text { Jacquinia aristata Jacq. } \\
\text { Cassia fistula L. } \\
\text { Capparis L } \\
\text { Crescentia cujete L. } \\
\text { Guazuma ulmifolia Lam. } \\
\text { Anacardium excelsum Skeels } \\
\text { Sterculia apetala (Jacq.) H.Karst. } \\
\text { Cordia dentata Poir. }\end{array}$ \\
\hline
\end{tabular}

Fuente: Elaboración propia a partir de trabajos de campo e IGAC (Estudio General de Suelos y Zonificación de Tierras. Dpto. de Bolívar). Nombre científico y siglas de los autores según "The International Plant Names Index". 
noviembre de 2010 y febrero de 2011 y únicamente fue encuestado personal adulto con edad mayor o igual a 18 años.

El cuestionario empleado constaba de 14 preguntas distribuidas en 4 sectores de indagación: datos generales o socio-demográficos, conocimiento de la vegetación de la zona, conocimiento sobre posibles beneficios o perjuicios provocados por las especies seleccionadas y diferenciación entre especies nativas y especies introducidas en el territorio de la CCV. El diligenciamiento de la encuesta también empleaba una guía con la imagen fotográfica de las especies en cuestión, en el entendido de que ello ayudaría a generar reminiscencias de experiencias pasadas y conocimientos previos, si fuese necesario (Williams \& Cary, 2002). Tal técnica ha sido empleada satisfactoriamente en estudios previos para identificar preferencias y actitudes humanas frente a especies en la modalidad de foto-cuestionario (Martín et al., 2007).

La percepción social fue evaluada en cuatro grupos sociales o actores de la trama social: (1) ciudadanía del asentamiento; (2) académicos o personal con formación científica relacionada con la conservación; (3) empresarios o habitantes que forman parte del sector productivo; y (4) miembros del gobierno o funcionarios del orden gubernamental con responsabilidad sobre la toma de decisiones en el sector medioambiental. La consideración mínima de respuesta tuvo en cuenta una escala valorativa del conocimiento y la percepción social de especies vegetales, cuyo umbral se situó en el $40 \%$ de los encuestados. Para establecer el número total de encuestas aplicadas entre los habitantes y la distribución porcentual de las mismas entre los distintos asentamientos integrantes del área de estudio, se tuvo en cuenta los datos poblacionales del Censo de 2005, así como una fórmula estadística y un muestreo que permiten seleccionar la muestra y establecer que ésta sea lo suficientemente representativa (Richardson, 1985; Martínez, 2003).

Para el caso que nos ocupa, se asumió que el territorio de referencia estaba ocupado por un total 1018578 habitantes (DANE, 2005), por lo que el número de encuestas pasadas a la ciudadanía, teniendo presente los redondeos necesarios, fue de 196. En cuanto al personal académico se aplicaron 24 encuestas a profesionales relacionados con la conservación; de igual manera se procedió con otros 20 empresarios o agentes activos del sector productivo; y ese mismo número de encuestas se pasó a funcionarios gubernamentales con responsabilidad en el área medioambiental con jurisdicción en la CCV. En total fueron 260 encuestas las que se distribuyeron para recoger la información de los distintos grupos sociales identificados previamente por el proyecto.

La totalidad de las encuestas se ejecutaron mediante entrevistas personales, y en ellas se valoró el impacto referido a una tercera parte de las especies reportadas como representativas del área de estudio (Tablas 2 y 3). Para seleccionar estas especies se consideraron factores de tipo ecológico, social y de gestión, de acuerdo con lo propuesto por García et al. (2008) en un estudio similar sobre percepción social en relación con el impacto de especies vegetales invasoras, procediendo según la experiencia recogida en el Estudio general de suelos y zonificación de tierras del Departamento de Bolívar (Instituto Geográfico Agustín Codazzi). Adicionalmente, se tuvo en cuenta si estas especies habían formado parte de programas de investigación, educación $o$ restauración implementados por entidades gubernamentales o instituciones ambientalistas. Y, por último, para la aplicación de los cuestionarios en el área específica del distrito de Cartagena, se tuvo en cuenta la división de los asentamientos distritales por Unidades Comuneras de Gobierno (UCG) y Localidades.

El nivel de percepción de las especies vegetales se tabuló según porcentajes, teniendo presente el total de encuestas aplicadas para cada uno de los grupos sociales identificados. Las respuestas al cuestionario fueron analizadas mediante técnicas estadísticas, estableciéndose las diferencias entre los grupos de interés encuestados y la actitud, percepción y nivel de conocimiento de la vegetación, mediante un análisis de varianza (ANOVA). Las diferencias que se encontraron en el análisis de varianza se dilucidaron mediante aplicación del Test de Tukey para rangos múltiples empleando el software Instat 3.

La valoración sobre el conocimiento y el estado de la percepción por parte de cado uno de los agentes sociales seleccionados, se efectuó teniendo en cuenta la siguiente tabla de escala valorativa, considerando un mínimo de $40 \%$ de los encuestados para estimar uso y preferencia (Tabla 4 ). 
Tabla 4. Escala valorativa del conocimiento y percepción social de especies vegetales presentes en la CCV.

\begin{tabular}{lr}
\hline Valoración & $\%$ Reportado en las encuestas \\
\hline Muy alta & $90 \%-100 \%$ \\
Alta & $70 \%-89.9 \%$ \\
Media & $40 \%-69.9 \%$ \\
Baja & $20 \%-39.9 \%$ \\
Muy baja & $0 \%-19.9 \%$ \\
\hline
\end{tabular}

Fuente: Elaboración propia

\section{Resultados.}

Conocimiento de la vegetación y percepción de beneficios.

La Tabla 5 nos muestra que, para un porcentaje $\geq$ $40 \%$ de los encuestados, los grupos sociales correspondientes a ciudadanía, gobierno y académicos reportan tener conocimiento de la existencia de las 12 especies vegetales sometidas a estudio, mientras que el grupo empresarios sólo identifican 7 de ellas, excluyendo a $P$. juliflora, $O$. wentiana, $P$. saman, $C$. pentandra, Guaiacum sp. En referencia a los aspectos de uso socioeconómico se encontró que la especie vegetal considerada de alta utilidad alimentaria para todos los grupos era C. nucifera, siendo llamativo el hecho que los grupos ciudadanía y académicos coincidan en reportar las especies C. uvifera, $C$. nucifera y $T$. catappa como de alta utilidad alimentaria.

Los beneficios ornamentales y paisajísticos están identificados en los cuatro (4) grupos sociales por $T$. catappa y por $C$. nucifera, esta última excluida únicamente por parte del grupo gubernativo. La utilidad medicinal está reportada mayormente y en todos los grupos por C. cujete. Nuevamente los grupos ciudadanía y académico coinciden al considerar a $C$. cujete y $G$. sepium como especies vegetales percibidas como de alta utilidad medicinal. Por su parte, la especie C. nucifera fue identificada por los encuestados como de gran interés comercial y económico en el caso de los empresarios, la ciudadanía y los académicos. Sin embargo, estos últimos son los únicos en catalogar a P. juliflora y O. wentiana como especies vegetales resistentes a altas temperaturas y escasez de lluvias. Esto resulta significativo teniendo en cuenta la condición representativa de estos vegetales como xerofíticos; mientras que los grupos ciudadanía, empresarios y gobierno coinciden en considerar para este aspecto a C. nucifera y T. catappa.
Conocimiento de la vegetación y percepción de perjuicios

Tanto el grupo empresarios como los encuestados del lote gubernamental coinciden en considerar a $A$. germinans y $R$. mangle como especies vegetales no deseables a la hora de tenerlas cerca de la casa o la calle donde habitan, en un porcentaje igual o superior al $40 \%$. En ese sentido, la Tabla 6 informa acerca de los posibles motivos de este rechazo, siendo los más esgrimidos por el segmento empresarial los referidos a posibles problemas derivados de los efectos del crecimiento de sus raíces (señalado por $70 \%$ de los encuestados); de servir de "refugio de delincuentes" (25\%); y de ser un "supuesto foco de enfermedades" $(25 \%)$. El rol gubernamental se expresa en términos muy parecidos, añadiendo un aspecto más, en este caso de carácter estético: el de "quitar vista a la casa" (25\% de los encuestados del grupo). No obstante, también se refirieron "otros motivos" como el mal olor, la presencia de espinas, la generación de suciedad por caída de hojas o ramas frágiles, la presencia de gusanos u hormigas o el ser potenciales conductores o llamadores de rayos, etc.

Conocimiento y percepción de la vegetación nativa y no nativa.

En la Figura 3 se recogen los datos acerca del conocimiento y la percepción de especies vegetales consideradas nativas en la CCV. A este respecto se aprecia que $C$. uvifera, $C$. nucifera, A. germinans y $R$. mangle, con un $95 \%$ de los reportes, son las especies con mayor registro de reconocimiento por parte del grupo de los empresarios. Esta misma es la consideración que hacen los grupos de gubernamentales y académicos. En concreto, este último grupo, coloca al más alto nivel de especies nativas a $G$. sepium y $C$. uvifera, con un $79.16 \%$ en ambos casos. En tanto que el colectivo ciudadanía entiende que $T$. catappa es una especie nativa en un $63.26 \%$.

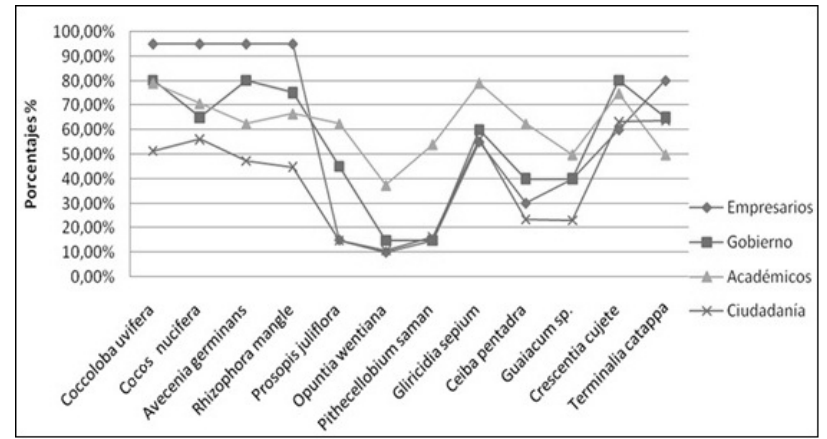

Figura 3. Conocimiento y percepción de especies vegetales consideradas nativas en la CCV por actor social. Fuente: elaboración propia. 
Estos resultados muestran que, según el grupo ciudadanía, las especies vegetales consideradas plenamente como nativas fueron $C$. uvifera, $C$. nucifera, G. sepium, C. cujete y T. catappa, siendo también a las que concedieron los mayores promedios de reconocimiento y percepción. ( $>50 \%)$. Por el contrario, la valoración más baja en cuanto a especies vegetales consideradas nativas se reportó para $P$. juliflora $(14,8 \%)$ y $O$. wentiana $(10,7 \%)$.

En lo que respecta al actor social empresarios, las especies vegetales consideradas como nativas fueron C. uvifera, C.nucifera, A. germinans, R. mangle, G. sepium, C. cujete y T.catappa, siendo asimismo las de mayores promedios de conocimiento y percepción

Tabla 5, Especies vegetales seleccionadas por los encuestados en cada grupo social, según el conocimiento de existencia, usos socioeconómicos y preferencias de especies.

\begin{tabular}{|c|c|c|c|c|c|c|c|c|}
\hline Actor Social & $\begin{array}{l}\text { Conocimiento } \\
\text { de la existencia } \\
\text { (a) }\end{array}$ & $\begin{array}{l}\text { Utilidad } \\
\text { alimentaria } \\
\text { (b) }\end{array}$ & $\begin{array}{c}\text { Beneficio } \\
\text { ornamental } \\
y \\
\text { paisajística } \\
\text { (b) }\end{array}$ & $\begin{array}{l}\text { beneficio } \\
\text { medicinal } \\
\text { (b) }\end{array}$ & $\begin{array}{l}\text { Interés } \\
\text { comercial y } \\
\text { económico } \\
\text { (b) }\end{array}$ & $\begin{array}{c}\text { Resistencia a } \\
\text { altas } \\
\text { temperaturas } \\
\text { y escasez de } \\
\text { lluvias (b) }\end{array}$ & $\begin{array}{c}\text { Deseable de } \\
\text { tener cerca } \\
\text { a la } \\
\text { vivienda o } \\
\text { calle (b) }\end{array}$ & $\begin{array}{c}\text { No deseable } \\
\text { de tener cerca } \\
\text { a la vivienda o } \\
\text { calle (b) }\end{array}$ \\
\hline Ciudadanía & $\begin{array}{l}\text { C. uvifera, } \\
\text { C. nucifera, } \\
\text { R. mangle, } \\
\text { A. germinans, } \\
\text { P. juliflora, } \\
\text { O. wentiana, } \\
\text { P. saman, } \\
\text { G. sepium, } \\
\text { C. pentandra, } \\
\text { Guaiacum sp., } \\
\text { C. cujete, } \\
\text { T. catappa }\end{array}$ & $\begin{array}{l}\text { C. uvifera } \\
\text { C. nucifera } \\
\text { T. catappa }\end{array}$ & $\begin{array}{l}\text { C. nucifera } \\
\text { T. catappa }\end{array}$ & $\begin{array}{l}\text { G. sepium } \\
\text { C. cujete }\end{array}$ & C. nucifera & $\begin{array}{l}\text { C. nucifera } \\
\text { C. cujete } \\
\text { T. catappa }\end{array}$ & T. catappa & \\
\hline Empresarios & $\begin{array}{l}\text { C. uvifera, } \\
\text { C. nucifera, } \\
\text { R. mangle, } \\
\text { A. germinans, } \\
\text { G. sepium, } \\
\text { C. cujete, } \\
\text { T. catappa }\end{array}$ & $\begin{array}{l}\text { C.nucifera, } \\
\text { T. catappa }\end{array}$ & $\begin{array}{l}\text { C. uvifera, } \\
\text { C. nucifera } \\
\text { T. catappa }\end{array}$ & $\begin{array}{l}\text { C. cujete } \\
\text { T. catappa }\end{array}$ & C. nucifera & $\begin{array}{l}\text { C. nucifera } \\
\text { T. catappa }\end{array}$ & $\begin{array}{l}\text { C. uvifera } \\
\text { C. nucifera }\end{array}$ & $\begin{array}{l}R . \text { mangle } \\
\text { A. germinans }\end{array}$ \\
\hline Gobierno & $\begin{array}{l}\text { C. uvifera, } \\
\text { C. nucifera, } \\
\text { R. mangle, } \\
\text { A. germinans, } \\
\text { P. juliflora, } \\
\text { O. wentiana, } \\
\text { P. saman, } \\
\text { G. sepium, } \\
\text { C. pentandra, } \\
\text { Guaiacum sp., } \\
\text { C. cujete, } \\
\text { T. catappa }\end{array}$ & C. nucifera & $\begin{array}{l}\text { Guaiacum } \\
\text { sp. } \\
\text { T. catappa }\end{array}$ & $\begin{array}{l}\text { G. sepium } \\
\text { C. cujete } \\
\text { T. catappa }\end{array}$ & $\begin{array}{l}\text { R. mangle, } \\
\text { P. saman } \\
\text { C. } \\
\text { pentandra } \\
\text { Guaiacum } \\
\text { sp. } \\
\text { C. cujete }\end{array}$ & $\begin{array}{l}\text { C. nucifera } \\
\text { C. pentandra } \\
\text { T. catappa }\end{array}$ & $\begin{array}{l}\text { C. nucifera } \\
\text { C.pentandra } \\
\text { Guaiacum } \\
\text { sp } \\
\text { T. catappa }\end{array}$ & $\begin{array}{l}R . \text { mangle } \\
\text { A. germinans }\end{array}$ \\
\hline Académicos & $\begin{array}{l}\text { C. uvifera, } \\
\text { C. nucifera, } \\
\text { R. mangle, } \\
\text { A. germinans, } \\
\text { P. juliflora, } \\
\text { O. wentiana, } \\
\text { P. saman, } \\
\text { G. sepium, } \\
\text { C. pentandra, } \\
\text { Guaiacum sp., } \\
\text { C. cujete, } \\
\text { T. catappa }\end{array}$ & $\begin{array}{l}\text { C. uvifera } \\
\text { C. nucifera } \\
\text { T. catappa }\end{array}$ & $\begin{array}{l}\text { C. uvifera, } \\
\text { C. nucifera } \\
\text { P. saman } \\
\text { Guaiacum } \\
\text { sp. } \\
\text { T. catappa }\end{array}$ & $\begin{array}{l}\text { G. sepium } \\
\text { C. cujete }\end{array}$ & $\begin{array}{l}\text { C. nucifera, } \\
\text { R. mangle, } \\
\text { A.germinans } \\
\text { C.pentandra } \\
\text { Guaiacum } \\
\text { sp. } \\
\text { C. cujete }\end{array}$ & $\begin{array}{l}\text { C. uvifera } \\
\text { C. nucifera } \\
P . \text { juliflora } \\
\text { O. wentiana } \\
\text { G. sepium } \\
\text { Guaiacum } \\
\text { sp. }\end{array}$ & $\begin{array}{l}\text { G. sepium } \\
\text { Guaiacum } \\
\text { sp. } \\
\text { T. catappa }\end{array}$ & \\
\hline
\end{tabular}

(a) Corresponde a las especies reportadas como existentes por cada grupo de análisis.

(b) Corresponde a las especies seleccionadas en porcentaje $\geq 40 \%$ de los encuestados en cada grupo de análisis. Fuente: Elaboración propia 
Tabla 6. Motivos por los que se rechazan especies que no se quieren tener cerca de casas o barrios.

\begin{tabular}{lllll}
\hline \multirow{2}{*}{ Motivos } & \multicolumn{2}{l}{ Empresarios (a) } & \multicolumn{2}{l}{ Gobierno (b) } \\
\cline { 2 - 5 } & Promedio & Varianza & Promedio & Varianza \\
\hline Refugio de delincuentes & 0.25 & 0.1973684 & 0.20 & 0.168421 \\
Posibles problemas por sus raíces & 0.70 & 0.2210526 & 0.55 & 0.2605263 \\
Supuesto foco de enfermedades & 0.25 & 0.1973684 & 0.05 & 0.05 \\
No son consideradas ornamentales & 0.05 & 0.05 & 0.15 & 0.1342105 \\
Quitan vista a la casa & 0.05 & 0.05 & 0.25 & 0.1973684 \\
Son de ramas frágiles & 0.05 & 0.05 & 0 & 0 \\
Otros motivos & 0.15 & 0.1342105 & 0.10 & 0.0947368
\end{tabular}

(a) Para el grupo empresarial el Valor F fue de 8.388 y su Valor crítico correspondió a 2.167 .

(b) Para el grupo gubernamental el Valor F fue de 5.118 y su Valor crítico correspondió a 2.167 .

Fuente: Elaboración propia.

$(60 \%)$. En este último sentido, los más bajos promedios de especies vegetales consideradas nativas se reportan para $P$. saman $(15 \%)$, P. juliflora $(15 \%)$ y $O$. wentiana $(10 \%)$, lo cual es coherente con lo recogido en la Tabla 5, donde el grupo empresarios no incluye estas especies como conocidas o percibidas en la zona de estudio.

A criterio del actor social gobierno las especies vegetales consideradas como nativas del territorio correspondieron igualmente a C. uvifera, C.nucifera, A. germinans, $R$. mangle, G. sepium, C. cujete y $T$. catappa, logrando promedios en conocimiento $\mathrm{y}$ percepción igual o superior al $60 \%$. Los más bajos porcentajes en cuanto a conocimiento y percepción de especies vegetales consideradas nativas los recogen $P$. saman $(15 \%)$ y $O$. wentiana $(15 \%)$. Para este actor social el conocimiento y la percepción de $P$. juliflora como especie nativa obtuvo una valoración media del $45 \%$.

Finalmente, para el grupo de académicos las diferencias entre las especies no son significantes en cuanto a conocimiento y percepción como nativa, aunque hay que señalar que los más altos promedios correspondieron a $C$. uvifera y $G$. sepium, ambas con un $79.17 \%$. Cabe resaltar, además, que para este actor social $O$. wentiana y T.catappa tienen los más bajos promedio entre el seriado de especies estudiadas, contrastando sobre todo T. catappa que muestra los menores niveles de percepción como especie nativa.

Por otra parte, al realizar el análisis de varianza (ANOVA), se constató que el valor estadístico $\mathrm{F}$, como estadístico de contraste en pruebas de comparación de varianza, indicaba, para el caso de los cuatro grupos de interés, resultados con valores significativos (probabilidad $\mathrm{P}<0.05$ ) para la gran mayoría de las especies vegetales analizadas, a excepción de G. sepium, C. cujete y T. catappa, las cuales no superaron el valor crítico para el valor estadístico F (2.639). El análisis ANOVA permitió comparar los distintos grupos, y las diferencias presentadas requirieron dilucidarse mediante un test de rangos múltiples (Test de Tukey). El procedimiento de Tukey mostró que para el caso de C. uvifera existen diferencias significativas entre los grupos ciudadanía y empresarios $(\mathrm{P}<0.001)$, así como entre ciudadanía y académicos $(\mathrm{P}<0.05)$. Se comprueba, así pues, que los grupos de empresarios y académicos consideran de forma significativa que $C$. uvifera es una especie nativa de la $\mathrm{CCV}$, si lo comparamos con el nivel de conocimiento o percepción reportado para el grupo ciudadanía. Para el caso de $C$. nucifera tal diferencia significativa sólo se presenta entre los grupo ciudadanía y empresarios $(\mathrm{P}<0.01)$.

En cuanto al grado de conocimiento y percepción de A. germinans y $R$. mangle como especies nativas de la $\mathrm{CCV}$, se encontraron diferencias significativas muy marcadas entre los colectivos ciudadanía y empresarios $(\mathrm{P}<0.001)$ y entre ciudadanía y gobierno $(\mathrm{P}<0.05)$, siendo el grupo ciudadanía el que reporta los menores promedios. Para el caso de P. juliflora se presentan diferencias significativas entre los actores sociales ciudadanía y gobierno $(\mathrm{P}<0.05)$, ciudadanía y académicos $(\mathrm{P}<0.001)$ y entre empresarios y académicos $(\mathrm{P}<0.001)$. De tal manera que se puede señalar que únicamente el grupo de académicos considera plenamente a $P$. juliflora como una especie vegetal nativa de la $\mathrm{CCV}$, siendo el de ciudadanía el grupo que menos la considera en tal sentido $(14,8 \%$ de percepción en promedio). O. wentiana, por su parte, mostró diferencias significativas entre los grupos ciudadanía y académicos $(\mathrm{P}<0.01)$, así como entre empresarios y académicos $(\mathrm{P}<0.05)$, lo cual indica que los grupos ciudadanía y empresarios no la consideran como especie nativa, siendo su valoración muy baja en cuanto a conocimiento y percepción $(10 \%)$.

Por último, la Figura 4 muestra cómo el mayor porcentaje de reportes, en tanto que especies vegetales consideradas extrañas a la $\mathrm{CCV}$, correspondió, para el caso del grupo ciudadanía, a $O$. wentiana $(35.2 \%)$ y a $P$. juliflora $(32.14 \%)$, que precisamente forman parte de la vegetación xerofítica del área de estudio. Aunque con bajo porcentaje de percepción y reconocimiento, $O$. wentiana constituye la especie considerada "no nativa" que registra los mayores porcentajes por parte de los grupos de gestores $(30 \%)$ y ciudadanía $(35,2 \%)$. En ninguno de los grupos sociales se perciben las especies valoradas como "no nativas" en porcentaje 
Ecol. apl. Vol. 13 № 2, pp. $97-108$

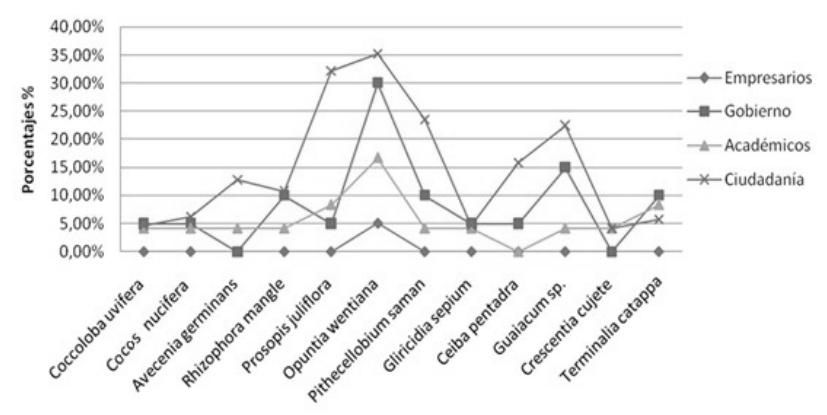

Figura 4. Conocimiento y percepción de especies vegetales consideradas no nativas en la región.

Fuente: Elaboración propia

igual o superior al $40 \%$ de los encuestados. Ahora bien, atendiendo al estadístico $\mathrm{F}$, el análisis ANOVA muestra valores significativos entre los diferentes grupos para el caso de $P$. juliflora, O. wentiana, $P$. saman, $C$. pentandra y Guaiacum sp, en las cuales se superó el valor crítico para el estadístico F (2.639).

\section{Discusión de los resultados.}

Las diferencias encontradas entre los distintos grupos sociales en cuanto a conocimiento, usos socioeconómicos y preferencias al seleccionar especies vegetales dependen, en última instancia, de las distintas formas de relación que establece cada grupo con el territorio, así como de sus intereses particulares. Ello coincide con lo expresado por Pírez (1995) en su trabajo sobre Actores sociales y gestión de la ciudad, donde señala que si bien los agentes sociales de un ámbito urbano dependen de procesos estructurales de tipo económico, socio-demográfico, político, etc., el papel que juega cada uno de ellos queda subordinado a la forma particular en que se organicen como unidades de acción, lo que, a su vez, es consecuencia de su relación con el territorio.

Al analizar la selección de especies vegetales que, en porcentaje mayor o igual al $40 \%$ de los encuestados en cada grupo de interés, fueron reportadas en aspectos relativos al conocimiento de su existencia, a los usos socioeconómicos y a la preferencia de especies, encontramos que el grupo de empresarios no reconoce $P$. juliflora, $O$. wentiana, $P$. saman, $C$. pentandra y Guaiacum sp., especies representantes de una vegetación xerofítica que no reviste interés comestible, medicinal o comercial para el grupo, lo que explicaría su aparente invisibilidad.

En cuanto a los usos socioeconómicos de las especies vegetales, se constató que el uso manifiesto de algunas de ellas como provisión alimentaria se centró, según todos los actores sociales, en C. nucifera (coco), mientras que los grupos ciudadanía y académico reportaron adicionalmente las especies $C$. uvifera (uvita de playa) y T. catappa (almendro). Con respecto a la utilidad medicinal, los resultados muestran que $C$. cujete, conocida comúnmente como "totumo", también es identificada por parte de todos los actores sociales de la CCV. Esto coincide con lo recogido en el trabajo que, a nivel nacional, realizo el Instituto de Investigación de Recursos Biológicos Alexander von Humboldt en 14 laboratorios naturistas, en relación a las plantas medicinales con mayor volumen de comercialización en el mercado nacional, y donde C. cujete era una de las más reconocidas. De igual manera, es importante señalar que el Instituto Nacional de Vigilancia de Medicamentos y Alimentos (INVIMA) de la República de Colombia ha incluido a $C$. cujete en el Vademécum de plantas medicinales, permitiendo el uso del jarabe fabricado con el extracto de la pulpa de su fruto como coadyuvante en el tratamiento de trastornos respiratorios leves (INVIMA, 2008). La encuesta nacional de plantas medicinales y aromáticas de Colombia, realizada en este caso por el citado Instituto von Humboldt y la Federación Naturista Colombiana (FENAT), muestra que las plantas medicinales son una parte fundamental del sistema de medicina tradicional y, al mismo tiempo, una importante fuente de ingresos para proveedores de materia prima $\mathrm{y}$ transformadores finales (Díaz, 2003).

Por otro lado, al contrario que el estudio del Instituto Von Humboldt, que no reporta a G. sepium (llamado popularmente "matarratón" en esta zona del Caribe colombiano) como una especie de interés medicinal de los laboratorios naturistas, nuestra investigación sí encontró que los grupos ciudadanía y académicos son coincidentes en considerar, además de C. cujete, la especie G. sepium como una planta de alta utilidad medicinal, especialmente aplicable en el tratamiento tópico de infecciones cutáneas como opción terapéutica no tradicional (Zuluaga et al., 2005). Además, en los últimos años, dicha especie ha venido utilizándose como suplemento de proteínas en el sistema de producción de rumiantes, cerdos e incluso aves, en ámbitos tropicales (González, 1996; Reverón et al., 1973). Se encuentra entonces un espacio para proyectar a Gliricida sepium como materia prima dentro del interés de los laboratorios naturistas del país, así como para valorar su uso dentro del sistema de medicina tradicional colombiana.

De otra parte, en el informe técnico sobre Caracterización del mercado colombiano de plantas medicinales y aromáticas, llevado a cabo asimismo por los citados Instituto Alexander von Humboldt y Ministerio de Ambiente, Vivienda y Desarrollo Territorial de Colombia, se estima que el mercado nacional de plantas útiles (medicinales, aromáticas y comestibles), tiene un valor entre 8 y 10 millones de 
dólares anuales, y se hace referencia a ventas cercanas al millón de dólares al año por parte de algunas de las empresas que fueron contactadas en el mencionado estudio. Entre las especies vegetales más significativas de este comercio destaca $C$. cujete (totumo).

Las especies xerofíticas sólo fueron percibidas de manera clara por el grupo académico, por lo que puede decirse que, en general, los habitantes de la CCV desconocen la importancia, por ejemplo, de leguminosas como $P$. juliflora, cuya siembra está siendo estimulada en lugares de baja productividad como el nordeste del Brasil, principalmente por su producción de vainas con miras a suplementar la alimentación animal en el período seco, y a cubrir la deficiencia nutricional de sus praderas. Se reporta, además, que el alto potencial del trupillo como especie vegetal útil en reforestación reside en sus características de precocidad, resistencia a la sequía y producción de madera de buena calidad para diversos fines. A la producción de vainas con elevada palatabilidad y alto valor nutritivo, se une la ventaja de fructificar en la época seca (Ribaski, 2000). De igual manera, en todos los grupos o actores sociales se reconoce $C$. nucifera como una especie de gran resistencia a altas temperaturas y a la indigencia pluviométrica.

Las diferencias entre el grupo académico y los demás actores sociales en relación a las especies resistentes a las altas temperaturas y escasez de lluvia, así como las distintas formas de valorar la utilidad alimentaria, medicinal u ornamental expresadas en la Tabla 5, pueden explicarse por la condición heterogénea del conocimiento local, tal como lo han expresado los trabajos de Gadgil et al. (2000), Brown (2003) o Ghimire et al. (2004), dado que, lo normal es que no todos los miembros de una comunidad tengan el mismo nivel de conocimiento ecológico. De igual modo, la reticencia a tener cerca ciertas especies vegetales, expresada tanto por empresarios como por gestores $(>40 \%$ de los encuestados), por ejemplo, frente a A. germinans (mangle negro) y $R$. mangle (mangle rojo), es una percepción coincidente con la detectada por estudios como el de Rodríguez et al. (2008), donde se muestra una tendencia nefasta para las zonas urbanas de manglar, evidenciando un descenso de los precios de las viviendas conforme se sitúan más cerca esta formación vegetal. Consecuentemente, los manglares urbanos son percibidos como zonas marginales y repulsivas de la ciudad, que ocasionan una depreciación de las propiedades. Esto termina constituyendo un acicate para su destrucción y su transformación en aras de un supuesto "mejor aprovechamiento", ya sea mediante la construcción de paseos peatonales, ampliando avenidas o abriendo zonas de parqueo. El subrepticio deseo de revalorizar las propiedades de finca raíz y el insaciable requerimiento de nuevos espacios urbanizables fomentan, en última instancia, una actitud negativa frente a la cercanía de estas especies, de lo que se deriva su sistemática eliminación, ya que, como indica Pírez (1995), "los actores económicos pueden incidir en el mercado local, y los actores políticos en las decisiones del gobierno local". No obstante, y aun siendo cierto que el grupo académico reporta interés económico para $R$. mangle, y que todos los grupos reconocen su presencia en el territorio, resulta llamativo que no se tenga conciencia de sus potenciales beneficios medicinales. Máxime cuando hay estudios que reseñan, por ejemplo, que el extracto acuoso de la corteza de $R$. mangle posee diversas propiedades farmacológicas, entre las que destacan su uso en el tratamiento de la mastitis bovina, la curación de heridas, las infecciones uterinas y las úlceras gastro-duodenales, beneficios todos ellos asociados a sus propiedades antisépticas, cicatrizantes, antiinflamatorias y antioxidantes (Sánchez et al., 2012).

Por su parte, el hecho de que el actor social ciudadanía considere a $O$. wentiana $(35.2 \%)$ y $P$. juliflora (32.14\%) como especies vegetales introducidas o extrañas a la CCV (Figuras 3 y 4), se correlaciona con la falta de información de una gran parte de la población, y pone de manifiesto nuevamente la condición heterogénea del acervo local. Los resultados muestran que varios de los grupos de encuestados consideran al almendro ( $T$. catappa) como una especie nativa de la zona, a pesar de ser originaria de Asia y haber sido introducida durante la época colonial, es decir, en un pasado lejano. Así lo creen, al menos, García et al. (2008), ya que afirman que la noción de "especie no nativa" es un concepto socialmente dinámico, y que tal reconocimiento se hace tanto menor cuanto más antigua es la introducción de la especie.

\section{Conclusiones.}

Nuestra investigación pone en evidencia que el conocimiento y la percepción acerca de las especies vegetales, así como en lo tocante a los servicios ecosistémicos con los que, eventualmente, determinadas formaciones vegetales puedan colaborar en el bienestar humano (agricultura, ganadería, comercio, medicina, ornato...), presentan notables diferencias según los distintos actores sociales. Se da el caso de que la mayor parte de la población de la CCV sólo percibe como especie de interés alimentario a $C$. nucifera, y como especies de utilidad medicinal a C. cujete y G. sepium, considerando, además, a $A$. germinans y $R$. mangle como especies indeseables (esta es precisamente la opinión más extendida entre empresarios y gestores, los dos colectivos más proclives a la eliminación del manglar).

Todo ello no es más que el reflejo de la diversidad de intereses, expectativas y valores de cada uno de estos grupos, así como de las relaciones de poder que dinamizan el entramado socio-ecológico de la CCV. 
De ahí la importancia de disponer de este tipo de información de cara al planeamiento participativo y al establecimiento de directrices para una gestión territorial coherente con el mantenimiento de la biodiversidad y los procesos naturales que la sostienen (Vilardy et al., 2012). Dada la significación territorial que adquiere este hecho, se vuelve prioritario promover vías de comunicación entre los actores sociales que fortalezcan la transferencia tanto del conocimiento formal como de los saberes tradicionales y su socialización. Con ello cabe esperar, en primer lugar, una reducción de la brecha existente entre el conocimiento de la población en su conjunto y el segmento académico (que es el que posee y puede generar y divulgar el conocimiento científico sobre los servicios que proporciona los ecosistemas sanos); y, en segundo término, un mayor acercamiento sinérgico entre empresarios y universitarios, algo más que deseable para la región, habida cuenta que, como ha demostrado Láscaris (2010), estos actores suelen tener una vinculación muy débil en América Latina.

En consecuencia, entendemos que el fomento de la educación y el reforzamiento de los vínculos existentes entre ésta, la investigación y la gestión, constituyen el motor que, junto a una activa participación ciudadana y un compromiso empresarial efectivo, permitirían, más allá de la mera pose nominalista, el desarrollo de una sociedad coherente con su sistema ecológico (Palomo et al., 2012).

\section{Agradecimientos.}

Los autores desean agradecer el apoyo de la Universidad de Cartagena (Colombia). A los ingenieros Luis Alberto García-Zapateiro y Alberto Gómez Juan por la colaboración en la sistematización y tabulación de la información; así como al colega Eduardo Caro Meléndez de Arizona State UniversityUSA por los apoyos de traducción. Es contribución al Grupo de Investigación RNM-273 del PAIDI y al CEIMAR (Comunidad Autónoma Andaluza, España).

\section{Literatura citada.}

Anderies J., Walker B. \& Kinzig A. 2006. Fifteen weddings and a funeral: Case studies and resilience-based management. Ecology and Society 11(1): 21. [online] URL:

http://www.ecologyandsociety.org/vol11/iss1/art21/ (Con acceso 23/07/2013).

Berkes F. \& Folke C. (eds.). 1998. Linking Social and Ecological Systems: Management Practices and Social Mechanisms for Building Resilience. Cambridge University Press, Cambridge, United Kingdom.

Borja F., Montes C. \& Román J.M. 2009. Regionalización Ecológica y Red de Espacios Naturales Protegidos de Andalucía. Proyecto Andalucía. Naturaleza XXXI. Ecología (IV):119-154. Publicaciones Comunitarias. Grupo Hércules. Sevilla. España.

Brown K. 2003. Three challenges for a real people-centered conservation. Global Ecology and Biogeography. 12: 89-92.
Callaghan P., Aznar P. \& Benayas J. 2004. Dimensión social en los proyectos de conservación de la biodiversidad. DEA. Doctorado Interuniversitario de Educación ambiental. UAM. España.

Capel H. 1973. Percepción del medio y comportamiento geográfico. Revista de Geografía. 1:58-150.

Conroy R. 2002. Lecture notes \# 1: perception \& cognition. [online] URL: http://undertow.arch.gatech. edu/homepages/rdalton/lectures/sc_01.htm. (Con acceso 23/07/2013).

DANE. 2005. Censo General. Nivel Nacional. Departamento Administrativo Nacional de Estadística. Colombia.

Díaz J.A. (ed.). 2003. Caracterización del mercado colombiano de plantas medicinales y aromáticas. Instituto Alexander von Humboldt y Ministerio de Ambiente, Vivienda y Desarrollo Territorial. Informe Técnico. Bogotá. Colombia.

Fernández Y. 2008 ¿Por qué estudiar las percepciones ambientales? Una revisión de la literatura mexicana con énfasis en Áreas Naturales Protegidas. Espiral, Estudios sobre Estado y Sociedad. XV (43). México.

Fleming D.K. 1975. What year is this? Yi-Fu Tuan. Topophilia. Annals of the Association of American Geographers. 65: 315-316.

Gadgil M., Seshagiri Rao P.R., Utkarsh G., Pramod P., Chatre A. \& Members of the People's Biodiversity Initiative. (2000). New meanings for old knowledge: The people's biodiversity registers program. Ecological Applications. 10: 1307-1317.

García M., Martín B., González J., Alcorlo P. \& Montes C. 2008. Social perceptions of the impacts and benefits of invasive alien species: Implications for management. Biological Conservation. 141: 2969-2983.

Ghimire S., McKey D. \& Aumeeruddy T. 2004. Heterogeneity in ethnoecological knowledge and management of medicinal plants in the Himalayas of Nepal: implications for conservation. Ecology and Society. 9 (3): 6.

Gibson J. 1980. El Campo Visual y el Mundo Visual. En: La Percepción del Mundo Visual. Páginas 47-68. Emecé. Buenos Aires. Argentina.

González M. 1996. Leguminosas forrajeras en sistemas de producción animal del Nor-Oriente de Venezuela. Leguminosas forrajeras Arbóreas en la Agricultura Tropical. Venezuela.

INVIMA. 2008. Vademécum Colombiano de Plantas Medicinales. Totumo. En: Plantas aprobadas por el Instituto Nacional de Vigilancia de Medicamentos y Alimentos. Imprenta Nacional de Colombia. Bogotá. Colombia.

Láscaris T. 2010. El Debate: capital social y efectividad de políticas tecnológicas en América Latina. Revista Iberoamericana de Ciencia, Tecnología y Sociedad. [online] URL: http://www.revistacts.net/elforo/362capital-social-y-efectividad-de-politicas-tecnologicasen-america-latina.

Lefebvre H. 1991. The production of space. Blackwell, Cambridge. United Kingdom

Lowenthal D. 1961. Geography, experience and imagination: towards a geographical epistemology. Annals of the Association of American Geographers. 51 (3):241-260 
Lynch K. 1960. The image of the city. Harvard University Press, USA (Trad. cast. de E. Luis Revol, La imagen de la ciudad, Buenos Aires. Ediciones Infinito, 1970).

Martínez C. (2003). Estadística y Muestreo. Décima tercera edición. ECOE. Ediciones Pedagógicas. Bogotá (Colombia).

Martin B., Montes C. \& Benayas J. 2007. The non-economic motives behind the willingness to pay for biodiversity conservation. Biological Conservation. 139: 67-82.

Matteucci S. \& Colma A. 1998. El papel de la vegetación como indicadora del ambiente. Páginas 293-320. En: Sistemas Ambientales Complejos: Herramientas de Análisis Espacial. Primera Edición. Eudeba. Centro de Estudios Avanzados. Universidad de Buenos Aires. Argentina.

Millennium Ecosystem Assessment. 2005. Ecosystems and Human Well-being. Synthesis. Island Press, Washington, DC. USA.

O'Brien K.L. \& Leichenko R.M. 2003. Winners and Losers in the context of global change. Annals of the Association of American Geographers. 93: 89-103.

Palomo I., Martín-López B., López C. \& Montes C. 2012. E1 sistema socio-ecológico de Doñana ante el cambio Global: Planificación de escenarios de Eco-futuros. Fundación González Bernáldez, Madrid. España.

Pírez P. 1995. Actores sociales y gestión de la ciudad. Revista Ciudades. 28:8 -14.

Quétier F., Tapella E., Conti G., Cáceres D. \& Díaz S. 2007. Servicios ecosistémicos y actores sociales. Aspectos conceptuales y metodológicos para un estudio interdisciplinario. Gaceta Ecológica. 84/85: 17-26.

Reverón A., Montilla J., Rodríguez J. \& Castillo P. 1973. Rabo de Ratón (Gliricidia sepium) una leguminosa del futuro en la alimentación animal. Rev. Pec. 340:25-29.

Ribaski J. 2000. Potencial del Algarrobo (Prosopis juliflora) en sistemas silvopastoriles en el semiárido de Brasil. Agroforestería para la producción animal en América Latina II. Depósito de documento FAO.
Richardson R.J. 1985. Pesquisa social: métodos e técnicas. Altas. São Paulo. Brasil.

Rodríguez M., Julio C., García A. \& de la Rosa M. 2008. Valoración económica de los manglares urbanos en la ciudad de Cartagena de Indias: Una aplicación a la gestión ambiental. Pontificia Universidad Javeriana. Facultad de Estudios Ambientales. Maestría en Gestión Ambiental. Cartagena. Colombia.

Sánchez J., Faure R. \& Mitjavila M.T. 2012. Efecto de Rhizophora mangle L. sobre la producción de anión superóxido en macrófagos marinos RAW 264.7. Rev. Cubana Plant. Med. 17(3): 223-232.

Torregroza E. (en preparación). Trama humana y componente natural en el ecosistema Ciénaga de la Virgen (Cartagena de indias - Colombia): Estrategias de restauración y adaptación. Tes. Doc. Universidad Internacional de Andalucía.

Torregroza, E., Hernández M., Barraza D., Gómez, A. \& Borja F. (en prensa). Unidades ecológicas para una gestión ecosistémica en el Distrito Cartagena de Indias (Colombia). Revista U.D.C.A Actualidad \& Divulgación Científica. Bogotá. Colombia.

Vilardy S., González J., Martín-López, B., Oteros-Rozas, E. y Montes, C. (2012). Los servicios de los ecosistemas de la Reserva de Biosfera Ciénaga Grande de Santa Marta. Revista Iberoamericana de Economía Ecológica. 19: 6683.

Warnock G.J. 1974. Philosophy of perception. Stroud, United Kingdom.

Williams K. \& Cary J. 2002. Landscape preferences, ecological quality and biodiversity protection. Environment and Behavior. 34(2): 257-274.

Zuluaga A., Upegui I., Rodriguez C.J., Ocampo M.C., Restrepo M., Parra G.J. \& Torres Y. 2005. Ensayo clínico fase I para evaluar la terapia con Gliricidia sepium en lesiones cutáneas de primates de la familia Cebidae. Cesmed. 19(1): 9-19.

1 Grupo de Investigación CTS. grupocts@gmail.com. Universidad de Cartagena (Colombia). Docente Investigador (Universidad de Cartagena, Colombia). E- mail: etorregrozaf@unicartagena.edu.co. Cartagena de Indias, Colombia. Formación: Licenciado en Biología (Universidad del Atlántico, Colombia), Especialista en Ciencia y tecnología de Alimentos (Universidad Nacional de Colombia, Sede Bogotá), Máster en Conservación y Gestión del Medio Natural (Universidad Internacional de Andalucía, España).

2 Grupo de Investigación CTS. grupocts@gmail.com. Universidad de Cartagena (Colombia). Docente Investigador (Universidad de Cartagena, Colombia). E-mail: jllamasc@unicartagena.edu.co. Cartagena de Indias, Colombia. Formación: Especialista en Teorías y Métodos de Investigación Social (Universidad de Cartagena, Colombia), Máster en Desarrollo Social (Universidad del Norte, Colombia).

${ }^{3}$ Universidad de Huelva (España). Profesor Titular de Universidad (Universidad de Huelva, España). Director de la Cátedra Doñana de Desarrollo Sostenible, Territorio y Energía (Eddea-Fundación Doñana 21. Universidad de Huelva). E- mail: fborja@uhu.es. Huelva, España. Formación: Doctor en Geografía Física (Universidad de Sevilla, España). 